\section{Association of Dermoscopic Profiles of Telangiectases with Nailfold Videocapillaroscopic Patterns in Patients with Systemic Sclerosis}

\section{To the Editor:}

The etiology of systemic sclerosis (SSc) remains elusive, but microangiopathy is considered to be significantly associated with disease pathophysiology ${ }^{1}$.

Morphological microvascular studies in patients with SSc use nailfold videocapillaroscopy (NVC) to examine the papillary capillaries in the nail bed, which run with their major axis parallel to the skin surface ${ }^{2}$ (Figure 1a). Cutaneous telangiectases (TA) are small enlarged end vessels, mainly permanent dilatation of postcapillary venules of the subpapillary plexus ${ }^{3}$.
They are readily visible; nevertheless, few studies have focused on cutaneous $\mathrm{TA}^{4}$.

Dermoscopy (DC) is a noninvasive technique used in the early diagnosis of melanoma $\mathrm{a}^{5}$ and nonmelanoma skin cancers, as well as inflammatory skin disorders (psoriasis, lichen planus, seborrheic dermatitis) ${ }^{6}$ The vascular structures of these conditions are detectable by DC, which has also provided data similar to NVC images commonly used in the study of nailfold capillaries in connective tissue diseases ${ }^{7}$.

In our study, DC was used to better appreciate vascular features of TA on selected anatomic sites (e.g., face, chest, hands, forearms) and to provide a morphological description of altered skin microcirculation in patients with SSc from a novel perspective. Using this approach, capillaries perpendicular to the skin, as well as the deeper portions of cutaneous

\section{(a)}

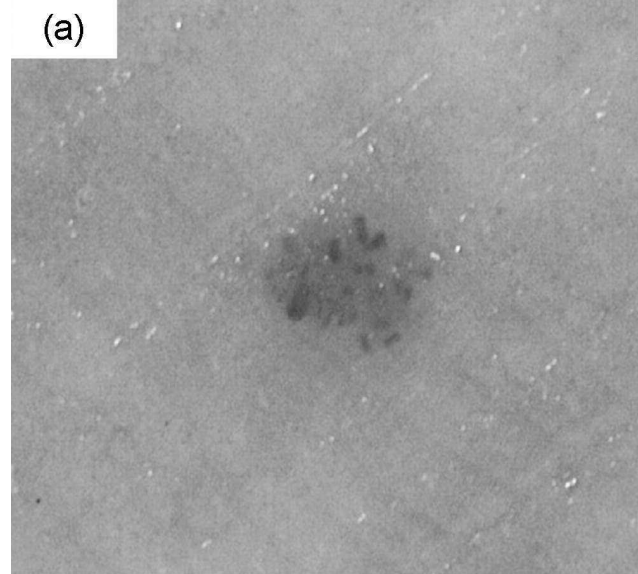

(c)

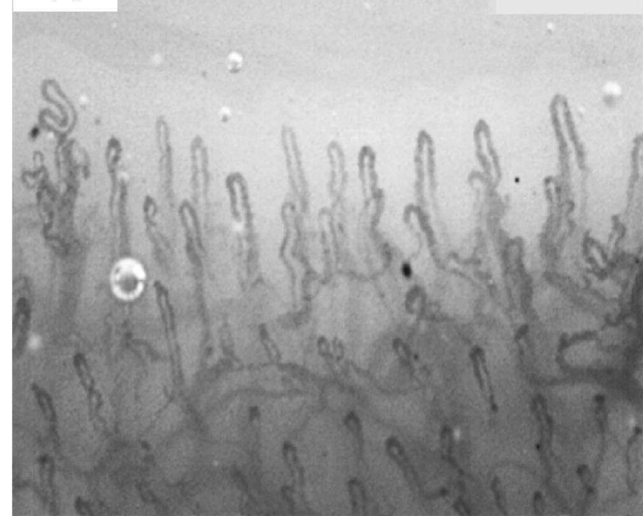

(e) Hemorrhages

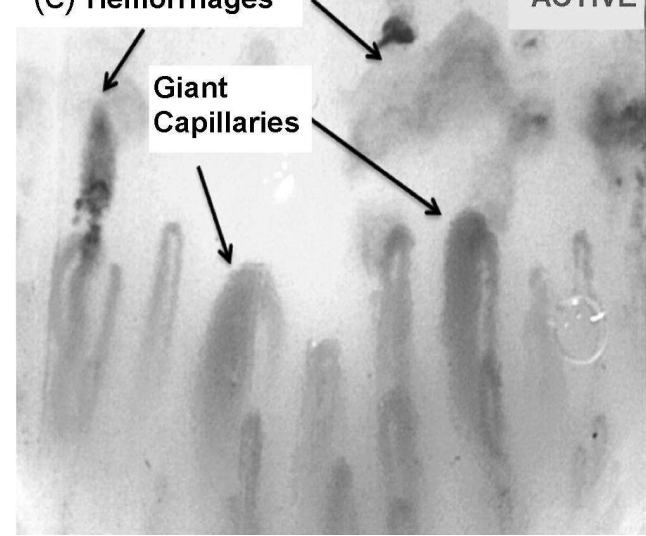

(b)

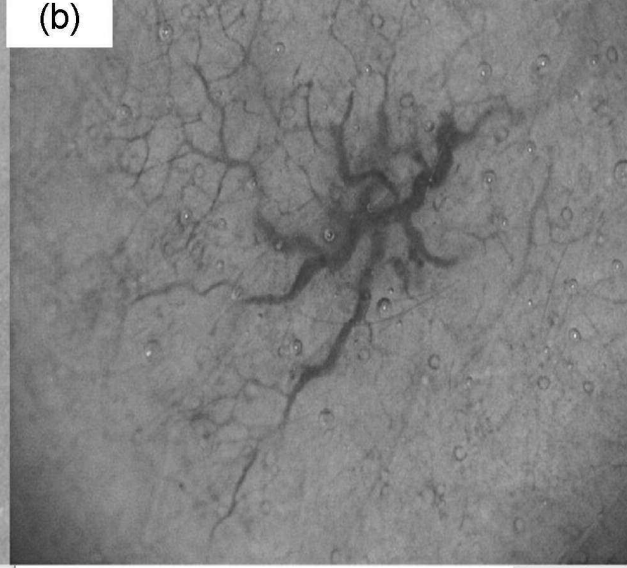

(d) Giant capillary with hemorrhage EARLY

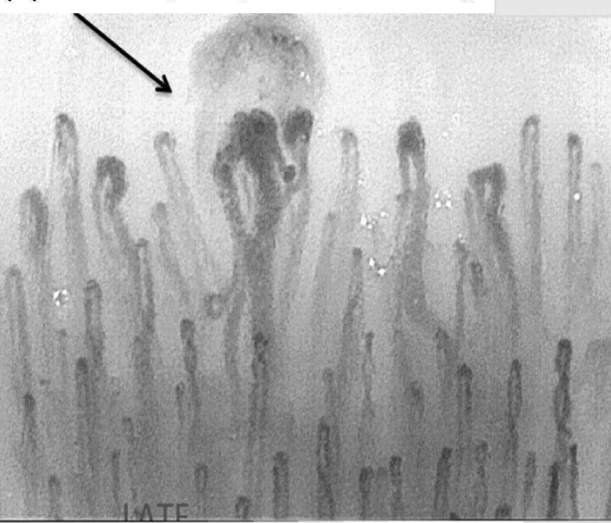

(f)

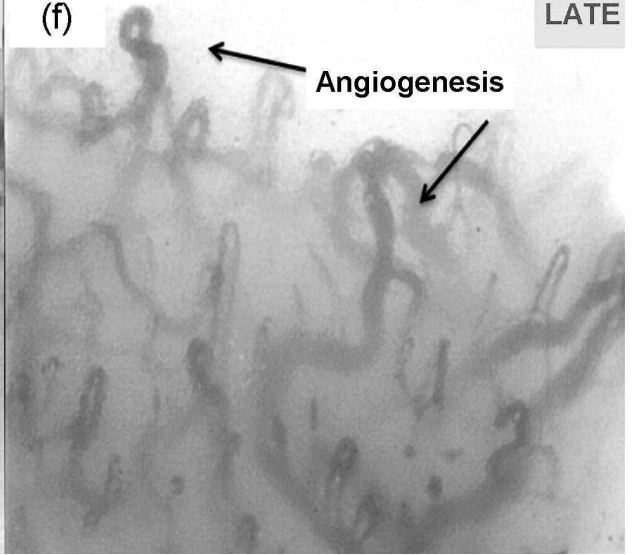

Figure 1. Dermoscopic spot (a) and reticular (b) telangiectases (TA) patterns are detected by using dermoscopy (DC). Nailfold papillary capillaries run parallel to the skin surface (c); in systemic sclerosis (SSc) their profile is altered and nailfold videocapillaroscopy (NVC) detects scleroderma pattern as early (d), active (e), and late (f) (200× magnification). In the other sites of the body (face, hands, chest), microvascular abnormalities may be revealed as TA (40× magnification; DB-Dermo Mips Leica $A G)$. 
microcirculation, usually not visible, become visible as they appear similar to TA.

DC patterns were investigated from 31 women with SSc (median age 55 yrs, median disease duration 8 yrs) showing skin TA and grouped into limited (1SSc) and diffuse ( $\mathrm{dSSc}) \mathrm{SSc}$-subtypes, according to clinical classification. They were investigated for antinuclear antibody (ANA) and specific antibodies, i.e., anticentromere (ACA) and antitopoisomerase (ATA), using standard immunofluorescence and ELISA methods. Patients were all women because of the high prevalence of this disease in this sex.

Two main DC patterns were found $(25 \times / 40 \times$ magnifications): a spot pattern (uniform rounded patches) and a reticular pattern (a reticular network; Figure 1a, 1b); they correspond to what Maricq, et al labelled as punctate and "mini-spider" using NVC on several areas of cutis and, more recently, others described clinically as matted and stellate TA, respectively 8,9 .

Based on the prevalence of one pattern to the other, a DC index was created, with $\geq 1$ indicating that the reticular DC pattern exceeds the spot pattern. The index was computed by calculating the difference between the number of reticular and spot DC lesions observed in each patient: briefly, an index $>0$ indicated that the reticular lesions outnumber the spot lesions.

We then investigated whether there was a relationship between the DC patterns of TA, NVC patterns (early, active, late) ${ }^{2}$ (Figure 1d, 1e, 1f), SSc clinical subtypes, and severity of disease (modified Medsger's index; Table 1). In a logistic regression analysis, a DC index $\geq 1$ compared to an index $\leq 0$ was found to be strongly associated with dSSc (adjusted OR 5.9, 95\% CI 0.9-38.5, p =0.06) and with the late NVC pattern (adjusted OR 13.2, 95\% CI 1.7-102.8, $\mathrm{p}=0.01$ ), independent of other variables shown in Table 1.

DC patterns of TA are related to the architecture of the cutaneous microcirculation under both normal and altered conditions ${ }^{10}$. The upper horizontal plexus occupies the papillary dermis and the most superficial part of reticular dermis, following an undulating path. Arterioles and accompanying venules join to each other forming a vascular network. From this tangled horizontal plexus, hairpin capillaries rise perpendicularly into the dermal papillae.

Hairpin capillaries run with the major axis parallel to the skin surface ${ }^{2}$ (Figure 1c) at the nailfold sites and at the mucous membranes, although they have a perpendicular array at other skin sites ${ }^{10}$. Thus, combining dermoscopic with NVC analysis would allow a more complete vision of SSc microangiopathy. This consists of progressive alteration and rarefaction of papillary capillaries (early, active NVC patterns) and further altered microvessels, ultimately leading to avascular areas (late NVC pattern). In SSc microangiopathy TA occur, consisting of ectasia of subpapillary plexus, detectable by DC, especially in dSSc TA.

We present for the first time preliminary cross-sectional data investigating in vivo SSc TA by ultrastructural, 3-dimensional, and qualitative descriptions of dilated postcapillary venules mainly in horizontal plexus ${ }^{10}$ and in papillary dermis ${ }^{2,3}$.

According to our results, DC is able to better identify alterations of the horizontal microvascular plexus (reticular DC TA pattern corresponds to the late NVC pattern) in patients with advanced SSc, whereas NVC allows the early detection of the papillary microvascular damage (early/active NVC pattern) only partially recognized by DC (spot DC TA pattern).

The index here, obtained by the qualitative DC analysis, may represent a potential clinical marker of disease severity, correlating with the other quantitative studies ${ }^{9}$. Further, it links the excess of reticular TA to diffuse dSSc and late NVC pattern. Indeed, patients with a positive DC index show a 6-fold risk of dSSc occurrence and a 13-fold risk of late NVC pattern occurrence (compared to patients with an index $\leq 0$ ).

This is the first study using dermoscopy to evaluate abnormalities of cutaneous microvessels in SSc TA and to investigate their relationship with NVC patterns.

ANNA R. GIAMPETRUZZI, MD, Dermoscopy and Nailfold Capillaroscopy Unit, Istituto Dermopatico dell'Immacolata, IRCCS; CHIARA MONDINO, MD, Department of Immunology and Allergology, Istituto Dermopatico dell'Immacolata, IRCCS; ANTONIO FACCHIANO, MD, Applied Dermatology Research Laboratory, Istituto Dermopatico dell'Immacolata, IRCCS; RICCARDO BONO, MD, Dermoscopy Unit, Istituto Dermopatico dell'Immacolata, IRCCS; PIETRO PUDDU, MD, Department of Immunology and Allergology, Istituto Dermopatico dell'Immacolata, IRCCS; BIAGIO DIDONA, MD, Department of Dermatology, Istituto Dermopatico dell'Immacolata, IRCCS; DAMIANO ABENI, MD, Health Services Research Unit, Istituto Dermopatico dell'Immacolata, IRCCS, Rome; MAURIZIO CUTOLO, MD, Research Laboratory and Academic Unit of Clinical Rheumatology,

Table 1. Percentage of patients in each level of each variable of interest within clinical subtype of scleroderma (SSc) and nailfold videocapillaroscopy (NCV) pattern.

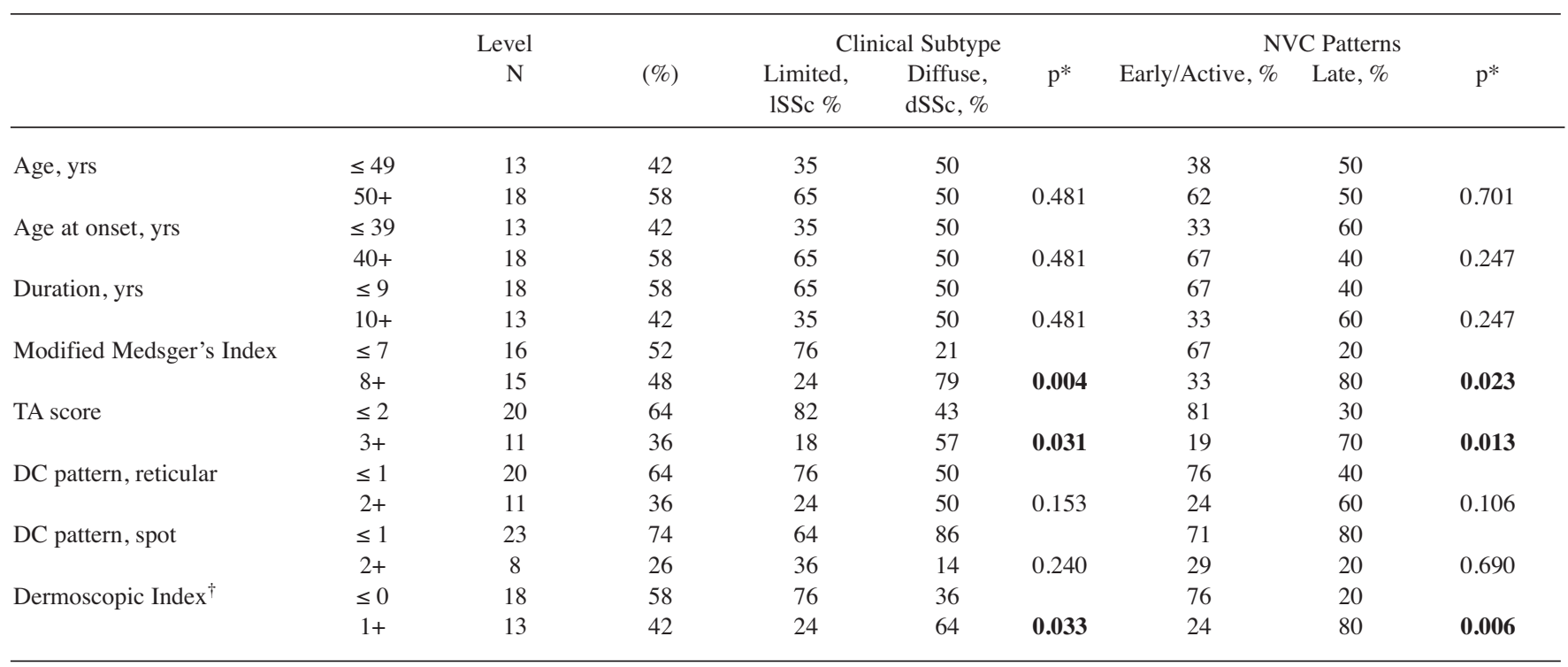

* Fisher's exact test, 2-tailed; $\mathrm{p}<0.05$ are in bold type. ${ }^{\dagger}$ Difference between the number of reticular and spot lesions. TA score: categorical score telangiectases; DC: dermoscopic.

Personal non-commercial use only. The Journal of Rheumatology Copyright @ 2013 . All rights reserved. 
Department of Internal Medicine, University of Genoa, Genoa, Italy. Address correspondence to Dr. M. Cutolo, Research Laboratories and U.O.C. Clinical Rheumatology, Department of Internal Medicine, IRCCS San Martino University Hospital, University of Genoa, Genoa, Italy.

E-mail:m.cutolo@unige.it

\section{REFERENCES}

1. Muller-Ladner U, Distler O, Ibba-Manneschi L, Neumann E, Gay $\mathrm{S}$. Mechanisms of vascular damage in systemic sclerosis. Autoimmunity 2009;42:587-95.

2. Cutolo M, Sulli A, Pizzorni C, Accardo S. Nailfold videocapillaroscopy assessment of microvascular damage in systemic sclerosis. J Rheumatol 2000;27:155-60.

3. Braverman IM, Ken Yen A. Ultrastructure and three-dimensional reconstruction of several macular and papular telangiectases. J Invest Dermatol 1983;81:489-97.

4. Murray A, Moore T, Herrick A. Clinical images: Systemic sclerosis-related telangiectases. Arthritis Rheum 2011;63:572.

5. Argenziano G, Soyer HP. Dermoscopy of pigmented skin lesions - A valuable tool for early diagnosis of melanoma. Lancet Oncol
2001;2:443-9.

6. Micali G, Lacarrubba F, Massimino D, Schwartz RA.

Dermatoscopy: Alternative uses in daily clinical practice. J Am Acad Dermatol 2011;64:1135-46.

7. Hasegawa M. Dermoscopy findings of nail fold capillaries in connective tissue diseases. J Dermatol 2011;38:66-70.

8. Maricq HR, Harper FE, Khan MM, Tan EM, LeRoy EC. Microvascular abnormalities as possible predictors of disease subsets in Raynaud phenomenon and early connective tissue disease. Clin Exp Rheumatol 1983;1:195-205.

9. Shah AA, Wigley FM, Hummers LK. Telangiectases in scleroderma: A potential clinical marker of pulmonary arterial hypertension. J Rheumatol 2010;37:98-104.

10. Braverman IM. Ultrastructure and organization of the cutaneous microvasculature in normal and pathologic states. J Invest Dermatol 1989;93 Suppl:2S-9S.

J Rheumatol 2013;40:9; doi:10.3899/jrheum.130171 